\title{
Négociations identitaires et comportements déviants à l'adolescence
}

Il est aujourd'hui admis que l'identité individuelle se construit durant la vie entière et qu'elle s'actualise dans chaque société. Elle commence au contact d'êtres proches et prend un essor tout particulier à l'adolescence où se négocie l'expérience objective et subjective dans une référence à l'histoire passée, au présent et à une vision d'avenir. Articulé autour d'éléments conscients et inconscients, le projet constitue dans nos régions une variable intermédiaire entre ces différents pôles car c'est autour de lui que s'organisent la personnalité en devenir, ses attitudes et ses valeurs. Dorénavant si les valeurs continuent à s'imposer quelque part aux personnes, elles ne sont ni transcendantales ni figées ni indépendantes des relations. C'est particulièrement vrai à l'adolescence.

Les médiateurs intervenant dans la construction identitaire et la négociation des valeurs se sont diversifiés et chacun peut s'accorder sur le fait que la culture renvoie désormais à quelque chose de mouvant et d'évolutif qui ne fait pas que se transmettre et qui de plus renvoie à bien d'autres lieux et institutions que la famille et l'école : les groupes de pairs, les médias et les visions du monde qu'ils dispensent, notamment en matière de consommation ou de rapport au travail. Parmi les processus décrits comme normaux dans la construction de l'identité à l'adolescence se trouvent le deuil de l'enfance, celui de l'infaillibilité des parents et du caractère illimité des éléments. Ces processus sont aujourd'hui décrits comme mis à mal par les évolutions sociétales. Par exemple, l'apprentissage de la maîtrise des pulsions et de la séparation entre fantasme et réalité deviendrait d'autant plus problématique que le fantasme devient une normalité 'regardable'.

Dans un contexte où les règles sont de moins en moins données d'avance et où les acteurs en place ont des difficultés à s'accorder sur la signification des situations et des rôles à tenir, l'identité devient un véritable travail et les choix, par leur caractère contraignant et indéfini, renforcent les enjeux. Se sont installés des espaces de 'marchandage' mais aussi de réelle négociation autour des valeurs. Ainsi, même si l'adolescence se prolonge de nos jours ainsi que les dépendances qui la caractérisent, on voit que les jeunes tentent de sortir de l'anonymat, se revendiquent de plus en plus tôt comme expérimentés, comme légitimes dans leur vécu, leurs idées. Dans ce mouvement, on remarque que les formes sociales d'expression ne sont plus monopolisées par les élites mais qu'elles se diversifient socialement et quant à leur contenu. Cela reste vrai même si, par ailleurs, les adolescents sont souvent présentés comme suivant les effets de mode tout en restant conventionnels au niveau des attentes et du classement des valeurs 'classiques'. On peut penser que les adolescents font émerger les contradictions et les peurs sociétales. Nombreux sont par exemple les jeunes qui ont intériorisé la 'culture' de la flexibilité, de l'éphémère et du risque mais leur apparente facilité à répondre à ces injonctions sociétales et à diversifier les modèles peut être interprétée de manière négative et frileuse par le monde adulte. Ainsi les problèmes intergénérationnels sont-ils loin de se limiter à une question d'incompréhension au sein de la sphère privée. Malgré son apparente 'émancipation', l'adolescent reste vulnérabilisé par le processus psychique en marche. Son expérience cognitive s'inscrit en lien étroit avec l'expérience affective. Alors que le conflit avec les parents lui permet d'introduire de la distance avec ces derniers tout en maintenant le lien, le jeune va trouver des modèles, des apaisements et des encouragements dans les contacts qu'il entretient avec d'autres jeunes, dans la fratrie ou auprès d'amis. L'adolescent se découvre potentiellement 'autonome' et 'puissant'. Sainsaulieu $(1985: 332)$ a démontré que l'exercice du pouvoir n'est pas anodin : il entraîne des conséquences culturelles sur le plan de l'identité, il est source d'un apprentissage de soi et de sa place dans le monde.

Pollack (1994) constate que l'identité devient préoccupation et objet d'analyse là où elle ne va plus de soi. Deux thèses s'affrontent quant à la racine du problème, la première voyant les acteurs comme devant affronter une situation de manque d'offre identitaire et la seconde les montrant comme oscillant entre différents pôles identitaires. L'association entre adolescence et délinquance juvénile, thème politique et social en vogue indépendamment de toute considération scientifique et statistique, illustre bien cette inquiétude et la confrontation entre différentes pistes explicatives.

\section{A. déficit d'offre identaire?}

Sceptiques quant au fait que le comportement déviant à l'adolescence proviendrait essentiellement de processus normaux (Born, 2005) et inquiets quant au durcissement de ses manifestations (vandalisme, 
toxicomanie, suicide), les observateurs qui soulignent les failles des procédures classiques de socialisation et de régulations sociales ne sont pas rares. Ils invoquent une crise morale et déplorent une démission de la famille, attribuant par exemple le fait que les jeunes s'installent dans une adolescence interminable aux ruptures identitaires et à l'absence de repères stables. L'opinion publique et les discussions politiques se nourrissent facilement de ce type d'explication. Néanmoins la recherche remet en cause le modèle évoqué de 'famille traditionnelle' et indique que si les rapports à la famille et à l'école sont bien au cœur des trajectoires déviantes, les relations entre le jeune, sa famille et l'école sont conjointement le baromètre de son comportement et sa résultante. De plus, tout parent peut constater que progressivement, il passe d'un rôle de coach à une position qui ressemble plus à celle de spectateur. Ainsi, le contrôle se révèle comme un exercice parental qui ne va pas de soi et qui, de plus, dépend de la situation matérielle et psychologique de la famille. En fait, ce ne sont pas seulement les interactions au sein des familles ou de l'école qui évoluent mais la place même de ces institutions dans le système social qui est ébranlée. Parmi les facteurs favorisant le passage à l'acte déviant, certaines recherches mettent bien en avant une massification de l'enseignement et une très grande difficulté de l'école à jouer son rôle d'égalisation des chances et de promotion sociale. A cela s'ajoute indéniablement des évolutions économiques et d'emploi laissant peu de place aux projets et aux capacités des groupes et familles les plus démunis. Le chômage des pères et la mise à mal de l'identité masculine populaire conduisent à une confiscation de la place de certains enfants qui en viennent à vouloir fuir une réalité identitaire peu supportable (par le suicide ou la dépendance aux produits par exemple). Dans ce schéma, la démission des parents ou l'autoritarisme exagéré constitue des réactions inefficaces mais qui relèvent en premier lieu d'une crise profonde de légitimité. Ce qui a changé ne serait pas l'ampleur des interdits mais leur légitimité. Ainsi, les activités d'apprentissage peuvent être vidées du sens et du 'droit' que le sujet a de s'y inscrire à un moment (Malewska-Peyre et Tap, 1991) et conduire à s'en désinvestir. Firchow (1992) présente la délinquance juvénile comme un terrain d'étude qui met également en évidence les problématiques de communication liées à la stigmatisation et à l'exclusion au sein du système-ville. Cette «excommunication » s'appuie sur une stigmatisation des familles, des parents et les enfants. Pour Dubet (2001), les banlieues françaises ne permettent plus la crise de l'adolescence. Maffessoli (2003) évoque quant à lui des rites de passage de plus en plus élitistes qui laissent dans le dénuement et l'exclusion une frange de plus en plus importante de la jeunesse. La réaction sociale formelle ou informelle est considérée comme une cause significative de la dyssocialité des adolescents. Or, selon Muchielli (2000), il semble devenu déplacé dans un contexte de néo-libéralisme de relier les problèmes sociaux et les inégalités sociales. Le conflit social et la notion même de conflit se retrouvent diabolisés alors que c'est un élément nécessaire de la constitution du lien familial et social, au risque de conduire à la violence. De la même façon, nos sociétés pousseraient à une confusion entre autorité et pouvoir. Or ce que les jeunes remettraient en cause, ce n'est pas la loi ou l'autorité en soi mais ce qu'ils ressentent comme de l'oppression. Ainsi, le jugement selon lequel les jeunes auraient désappris les règles de la citoyenneté occulte la question du pouvoir et peut déboucher sur une internalisation des causes. Parfois, pour contrer le sentiment d'impuissance sociale devant les inégalités du réel, prendre une place négative par la déviance développée vaut mieux que de ne pas avoir de place du tout. Dès lors, cette réaction ne peut plus être seulement considérée un problème individuel mais comme un élément participant à une idéologie sociétaire. Au bout du compte, même si le phénomène de délinquance juvénile reste limité et prend des formes variées, il renvoie à l'expérience commune de souffrance. Différents réseaux peuvent entrer en compétition dans la recherche d'une solution, d'un dérivatif. Les conversions se placent le plus souvent à des moments de fragilité psychologique. Sachant que l'immigration et sa stigmatisation viennent se surajouter aux problèmes identitaires et d'exclusion, l'affirmation d'une identité religieuse peut prendre place dans cette dynamique. Elle est à comprendre comme une des manières de redonner sens à sa vie, de rétablir le lien avec 'sa' communauté. Elle réorganise les conduites, rend du sens et sécurise car elle n'autorise aucun doute de par la vision dichotomique et essentialiste du monde et du bien et du mal qui l'accompagne.

De son côté, la bande de copains est une solution alternative à l'entrée dans la vie active. Elle permet d'investir de nouveaux espaces et repères, d'acquérir un sentiment de maîtrise. Les interactions qui se poursuivent sont alors plus à voir comme une adaptation et une socialisation réciproque. Leur compréhension exige de prendre en compte l'ensemble des acteurs en présence, ce qui fait dire que 
plutôt que d'étudier les causes da la délinquance, l'enjeu consiste à étudier les vies des jeunes dans leurs bifurcations et leurs interactions réelles, spatialement et temporellement situées.

Ainsi, si l'individu est un acteur et même s'il est conditionné par les structures, il n'en subit pas seulement les effets. On voit émerger à la fois des configurations particulières et des logiques récurrentes d'action, le comportement déviant se confirmant comme une des formes d'expérimentation identitaire. Aujourd'hui, plus qu'avant, il existe un consensus des chercheurs à comprendre la logique des acteurs et les moyens qu'ils mobilisent, cela sachant que les situations, sentiments et comportements peuvent évoluer au cours du temps dans les deux sens. Les spécialistes contemporains de l'adolescence prônent pour la plupart une vision interactionniste, à la croisée des racines bio-sociopsycho-culturelles du comportement et du jeu des structures. Pour N. Elias (1991), l'opposition entre ceux qui axent leur attention sur les facteurs individuels et ceux qui axent cette dernière sur les facteurs sociaux n'a en fait rien de réel mais elle ne s'explique qu'en fonction de deux traditions politico-philosophiques erronées. J.C. Kaufmann (2001) en insistant sur le rôle joué par les interactions quotidiennes et les habitudes, invite à approcher les parcours et les contextes sociaux localisés où jouent conjointement les relations avec les familles, les quartiers, les groupes de pairs et l'ensemble des institutions. Plusieurs auteurs s'accordent pour penser que la dialectique entre structures sociales médiatisées par des interactions concrètes et la formation d'un soi individuel s'avère constante et que ces interactions ne sont pas unilatérales. Ainsi, au sein des échanges intergénérationnels, il arrive qu'un adolescent puisse rendre un peu d'autorité au parent qui s'en sent dépossédé. Par ailleurs, il n'est pas rare que les régulations institutionnelles débouchent sur des effets contradictoires et complémentaires : tantôt l'action éducative peut renforcer l'identité stigmatisée du jeune 'à problèmes', tantôt elle va être perçue par le jeune comme un appel positif à rejoindre la société. Au bout du compte, le processus en jeu au cœur des trajectoires et transactions biographiques n'est pas seulement individuel mais représente bien un fait social significatif.

\section{B. Oscillation entre différents pôles identitaires?}

. Les problèmes identitaires et les comportements délinquants doivent toujours être replacés dans une vision dynamique où l'individu fixe ses choix d'appartenance grâce à ses interactions avec les autres individus ou groupes. Il y a une participation active de l'individu à rechercher ce qui est cohérent et significatif pour lui dans son univers quotidien et qui lutte pour la reconnaissance de la part des groupes auxquels il appartient. L'accent est mis ici sur la concurrence entre plusieurs appartenances, voire leur incompatibilité. La culture devient ici le foyer symbolique par excellence (Coslin, 2007) car c'est là que les individus viennent puiser ce qu'ils jugent significatif pour eux, pour leur identité. Camilleri (1990) s'est intéressé aux stratégies identitaires des jeunes pour faire face aux conflits et à leurs échecs éventuels. Il met l'accent sur le fait qu'il y a négociation constante des appartenances en termes de rapports de rapprochement fondés sur les similitudes, et d'éloignement fondés sur les différences. Le jeune doit s'orienter dans des choix de valeurs et de projets de vie parfois rendus difficiles en raison de références culturelles contradictoires. De nombreuses études ont porté durant les dernières décennies sur les jeunes immigrés de "deuxième génération » présentés comme tiraillés entre modernité et tradition. Mais ce dont on se rend compte aujourd'hui, c'est que ce qui différencie les jeunes allochtones et autochtones au niveau identitaire n'est en fait pas tant leur différence culturelle mais le regard qu'ils portent sur les cultures plurielles auxquelles ils appartiennent et en articulation desquelles ils se définissent et ils négocient leurs représentations d'eux-mêmes. Pour Tichit (2005), les positionnements individuels vont à l'encontre d'une vision trop mécanique de l'intégration comme une vision trop homogénéisante des catégorisations ethniques utilisées dans les politiques publiques qui privilégient la seule facette de la désignation, sans prendre en compte l'autre facette de l'autodéfinition. Celle-ci met à jour le choix individuel de chaque acteur social face à son altérité et les multiples façons sur un continuum large de se définir autrement. JF Bayart (1996) parle à ce propos de fausse illusion identitaire. Le fait que ces jeunes sont considérés comme déracinés, confrontés à une crise ou des écarts d'identité permet aussi de faire l'économie d'une réflexion en terme de rapports sociaux pour expliquer les expressions violentes des jeunes immigrés. Une approche en termes de pluri-identité rend très difficile une réflexion en termes de discrimination institutionnelle, renforçant une racialisation des lectures du monde. Un lien est peut-être à faire avec une ethnicisation des rapports entre élèves au sein des écoles et une multiplication des insultes racistes, cela 
principalement au sein des populations assujetties à une triple pression structurellement ancrée (économique, raciale et sexuée). Selon Franchi (2002), le recours à des insultes racistes pourrait être une stratégie identitaire des jeunes qui cherchent à se protéger eux-mêmes des méfaits du racisme ambiant en se désaffiliant d'un groupe construit comme homogène par la société et en disqualifiant l'autre par un discours qui vise à pointer son altérité et infériorité. Selon l'auteur, une explication de la réalité des jeunes dans des termes de différence culturelle court le risque de gommer l'existence de rapports de force au sein même des cultures minoritaires, dont par exemple, les violences subies par les jeunes filles en situation d'asymétrie socio-économique, et de légitimer encore plus le construit de l'ethnicité.

En fait, nombreuses sont les situations où les jeunes, et pas seulement les jeunes allochtones, se retrouvent écartelés dans leur construction identitaire. Pour Qribi (2005), les conditions sociales doivent être articulées avec la manière dont les sujets s'approprient le social et le culturel. On découvre alors une jeunesse aux prises avec des références contradictoires et un vécu qui l'est tout autant. Ainsi, pour les jeunes en rue, cette expérience peut représenter à la fois une menace et un espace propice à leur développement. On peut par ailleurs observer que les jeunes gens et les jeunes filles se retrouvent aussi 'tiraillés' en fonction de référents et enjeux spécifiques à leur groupe sexué (Gavray, 2004), ce qui renvoie à la notion d'ambivalence développée par Kaufmann (2001). A propos des jeunes filles, une socialisation contradictoire découlerait de la difficulté à unifier une identité identifiée comme 'féminine' axée sur la famille et une identité axée sur le travail. Cette difficulté découlerait de deux dynamiques historiques spécifiques et concurrentes: d'un côté une mémoire longue de schèmes genrés et de l'autre un emballement récent des trajectoires d'autonomie féminine. De son côté, Commaille (2002) accorde une grande place à la manière dont les hommes et les femmes mêlent vie privée et vie publique et concilient leurs identités. Touraine (1997) confirme de son côté que le défi tant individuel que familial et sociétal contemporain réside surtout autour d'enjeux de proximité, dans la nécessité de ré-harmoniser des sphères éclatées ou en compétition, dans la mise en lumière des pratiques innovantes qui rendent compatibles l'instrumentalité et l'identité, deux univers que la modernisation a séparées. Notons que la référence subjective à l'emploi, producteur de sens même s'il faut le désacraliser, lui semble, comme à de nombreux analystes, incontournable, cela indépendamment du contexte de fragilisation et de pénurie qui l'affecte et de l'univers masculin qui l'imprègne. Duret (2001) affirme de son côté que la construction identitaire de genre devient de plus en plus complexe alors que les images stéréotypées du point de vue du sexe restent bien présentes dans la population et dans l'élaboration des connaissances. Cet état de fait peut expliquer l'incompréhension quant à l'évolution des problèmes de comportement et de déviance des filles. Selon Tichit (2005), la délinquance continue à être d'abord lue en termes d'inadaptations témoignant d'arguments de nature et de problèmes psychologiques des filles alors que pour les garçons, les études mettent l'accent sur les facteurs extrinsèques : les pairs, les relations interpersonnelles, les éléments institutionnels. La difficulté de considérer les filles comme des individus singuliers, et non comme un groupe 'homogène' par nature, ainsi que la résistance à interroger la place sociale qui leur est octroyée dans les groupes minoritaires, aident aussi à comprendre l'absence de questionnement sur l'ethnicité à propos des filles. Manço (1999) montre en quoi les jeunes filles se retrouvent particulièrement au cœur des enjeux d'interculturalité. Parvenir à négocier son être, son identité entre deux cultures relève pour elles d'une mobilisation particulière de toutes les ressources et de tous les acteurs en présence. Les jeunes filles, très sensibles à leur reconnaissance sociale dans le pays d'accueil, utilisent et articulent différentes stratégies: d'évitement, de contournement, d'affrontement et de surenchère. Pour ce chercheur, l'efficacité de la gestion des contradictions symboliques semble liée à l'appropriation personnalisée et critique des valeurs. Des difficultés peuvent entraver le processus, engendrant des situations conflictuelles, violentes et les femmes de toutes les générations n'ont pas les mêmes ressources et possibilités que les hommes pour prendre part au processus. La plupart des témoins de la situation des femmes immigrées sont des femmes qui font souvent le constat d'interventions institutionnelles peu adaptées.

Néanmoins, le rôle des intervenants se révèle aujourd'hui important dans la résolution des situations problématiques impliquant des jeunes comme dans l'aide apportée à ces derniers dans leur lutte pour la reconnaissance. Accompagner le jeune devient aujourd'hui 'l'épauler et ne pas prendre sa place' ; c'est aussi inciter à transformer son regard pour qu'un problème devienne plutôt une étape à intégrer 
dans un parcours dont on tente d'aider à amorcer au mieux les passages. Les interlocuteurs naturels des ados (enseignants, animateurs) sont mobilisés dans le but de rétablir une vraie communication, condition et outil évalués de nos jours comme très importants pour prévenir la délinquance juvénile. Bordes (2007) décrit un processus de socialisation réciproque entre les jeunes et certaines institutions locales ou municipalités où le pouvoir intervient comme un opérateur retravaillant les différentes identités (relationnelles, narratives et argumentatives) et où régulations de pouvoir et de savoir se nourrissent l'une de l'autre. La socialisation devient alors un vrai chantier innovant et émergeant construit par une jeunesse contrainte à se battre, y compris avec ses paradoxes. C'est par exemple le cas autour du développement du rap ou d'autres mouvements musicaux et culturels : la municipalité peut fournir un cadre et la scène institutionnelle aide à développer la maîtrise des jeunes. La négociation permanente des espaces que ces derniers provoquent leur permet de se construire et de se réaliser comme acteur social. De plus, ils provoquent des mutations dans un fonctionnement conçu au départ par des adultes loin des préoccupations des jeunes Cette négociation débute au niveau local mais peut prendre de la hauteur. Cet exemple peut probablement se comprendre comme l'illustration de la diversification et de la généralisation de la négociation évoquée par $O$. Kuty.

\section{Bibliographie}

Bayart J.F., 1996, l'illusion identitaire, Paris, Fayard.

Bordes V., 2007, Prendre place dans la ville. Jeunes et politiques municipales, Paris, L'Harmattan, Collection Débats Jeunesse.

Born M., 2005, Psychologie de la Délinquance, Bruxelles, De Boeck Université.

Camilleri C. et al. 1990, Stratégies identitaires, Paris, PUF.

Commaille J., 2002, La politique de la famille, Paris, La découverte.

Coslin P., 2007, La socialisation de l'adolescent, A. Colin.

Dubet F., 2001, Les inégalités multipliées, Editions de l'Aube.

Duret P, 2001, "On ne naît pas homme, on le devient », dossier : les hommes en question, Sciences humaines, 112, pp.32-35.

Elias N, 1991, La société des individus, Paris, A. Fayard

Franchi V., 2002, «Ethnicisation des rapports entre élèves. Une approche identitaire, Ville-EcoleIntégration ", Enjeux, Hors Série ${ }^{\circ} 6$.

Gavray, C., 2004, «Engagements familiaux et professionnels : regard sur les projets des jeunes hommes et femmes en fin de formation scolaire », in Istace E., Laffut, M., Plasman, R, et Ruyters, C., Sphères privées et professionnelles : vers une recomposition des rôles et des actions, de Boeck, coll. Economie, Société et Région, $3^{\text {ème }}$ partie, ch. 7, pp 199-220.

Kaufmann J.C., 2001, Ego. Pour une sociologie de l'Individu. Une autre vision de l'homme et de la construction du sujet, Paris, Nathan.

Maffesoli M., 2003, Notes sur la postmodernité, Félin.

Malewska-Peyra H. et Tap P. (éds), 1991, La socialisation de l'enfance à l'adolescence, Paris, PUF.

Mohamed A., 2001, «Vivre son adolescence à la croisée entre deux cultures : entre crise identitaire, rupture, délinquance et galère ? », VEI Enjeux, 126, pp.166-186.

Manço A., 1999, Intégration et identité. Stratégies et positions des jeunes issus de l'immigration, Bruxelles, De Boek Université.

Muchielli L., 2000, "Le contrôle parental du risque de délinquance juvénile: un bilan des recherches », Les cahiers de la sécurité intérieure, 42, pp.127-146.

Pollack M., 1994, Une identité blessée, Paris, Métailié.

Qribi A., 2005, «Emeutes urbaines : les raisons de la colère », Lien Social, 776, pp.8-9.

Sainsaulieu R., 1985, L'identité au travail, Paris, Presses de la Fondation Nationales des Sciences Politiques.

Tichit L., 2005, «Rapports sociaux de genre et construits ethniques : l'exemple des gangs de filles des minorités à Montréal », in Queloz N. et al. (dir), Délinquance des jeunes et justice des mineurs. Les défis des migrations et de la pluralité ethnique, Berne, Stampfli, pp.173-184.

Touraine, 1997, Le monde des femmes, Fayard. 
Born Michel, Professeur Ordinaire, Faculté de psychologie et des sciences de l'éducation, Ecole de Criminologie

Gavray Claire, Première Assistante et Chargée de Cours adjoint, ISHS et Faculté de Psychologie, département 'Personne et Société'

Adresse des auteurs : Université de Liège B33 Sart-Tilman 4000 Liège tél 043662272 